\title{
Who Poisoned Hugh? - The STAR Framework: Integrating Learning Objectives with Storytelling
}

\author{
Andreea Molnar ${ }^{1}$, David Farrell ${ }^{2}$, Patty Kostova ${ }^{1}$ \\ City eHealth Research Centre, City University London, UK ${ }^{1}$ \\ Glasgow Caledonian University, $\mathrm{UK}^{2}$ \\ Andrea.Molnar.1@city.ac.uk, david.farrell@gcu.ac.uk, \\ pattyesoi.city.ac.uk
}

\begin{abstract}
Little research in Interactive Digital Storytelling (IDS) has been given to writing stories that convey a set of Learning Objectives (LOs). This is particularly important for educational IDS games. In this paper we propose the STAR framework for formalizing the design of IDS stories for educational interventions. The story is designed as a set of red-herrings and clue puzzle in which the LOs are integrated. We present one of the three IDSes we designed based on this framework; we use one as a case study and present its evaluation. A study was undertaken to evaluate the game effectiveness in conveying the educational message. This study performed with 145 players shows that the players' knowledge improved as a result of playing the game and that the change was statistically significant.
\end{abstract}

Keywords: interactive digital storytelling, serious games design, narratives, game design, game based learning

\section{Introduction}

Interactive Digital Storytelling (IDS) games have shown positive results when used for educational purposes [1]. However, there is little available out there "on how to design and facilitate games for learning" [2]. This research proposes to add to work on educational games design by focusing on storytelling within games [3], when they are used for educational interventions. Although in this arena, there is some research on how to design the stories for entertainment purposes [4-7] little attention has been given to stories designed specifically for educational interventions. This research contributes to the work in this arena, by showing how the Learning Objectives (LOs) could be conveyed through an engaging plot. Our aim is to propose a framework for designing an interactive story so that the essential LOs are covered. Each story is designed as a set of puzzles and each puzzle contains different LOs. We use the framework to design three different IDSes and evaluate them.

We present as a case study the Global Handwashing Day (GHD) game. The way we constructed the puzzles and we integrated the LOs in the puzzle are also presented. 
The rest of this paper organized as follows. Section 2 presents the existing work in the area. Section 3 presents our proposed framework STorytelling for educAtional inteRventions (STAR). Section 4 introduces the three games we created using the proposed framework and presents in detail the Global Handwashing Day (GHD) Game design. Section 5 presents the evaluation and the future work. Section 6 ends the paper with our conclusions.

\section{Background and Related Work}

Research studies on IDS have focused on different aspects of the design. Among them, [4] proposes a pattern library for role playing games "to better understand the relationship between level and quest design". [5] proposes to use techniques from cinema, literature and gaming world to generate IDS. [6] proposes to design interactive narratives around the idea of roles and functions. [7] designs the game using the following theories: narrative-centered learning, activity theory and cognitive load theory. [8] performs a preliminary study to assess the user experience when the game is text based or delivered through the graphical interface. The result of the study shows the advantages and disadvantages of each of the version, with graphical version being more appealing and easier to navigate, while the text based stimulating the imagination more than the graphical one. Our approach differentiates from the previous approaches mainly by focusing on IDSes aimed at educational interventions.

In this area there is little guidance for game designers to design educational games, and little research to assess what is effective in educational games [2]. Among the few researchers looking at this problem, [9] looks at whether or not adding narratives to adventure computer games improve the academic learning content and learner enjoyment. They find that the addition of narratives does not significantly enhance the learning of educational content. Concerning the learner enjoyment, participants who played the game enhanced with the narratives enjoyed the game slightly more, but this difference was not statistically significant.

Our approach differentiates from the current approach by taking a slightly different path. First we focus mostly on story generation, for narrative educational games. We show how we designed the story such that we obtain a statistically significant improvement in student knowledge. Although the research of [9] shows that narratives do not add value to games, our approach is differentiated from theirs by the fact that we include the educational content in the narrative, whereas the [9] approach added the narratives just as a support to the game, and the educational content was not integrated into the story. This approach has been shown not suitable for educational games, as the players pay more attention to the story and ignore the educational content that is being taught [10].

\section{Educating by Storytelling: the STAR Framework}

The requirements for STorytelling for educAtional inteRventions (STAR) framework are: 
1. Engaging story plot

2. Convey a given set of LOs (with the options of varying educational importance)

3. Reinforce important LOs

4. Flexibility in adding or removing LOs as required

5. Interactivity by allowing the user to influence the story

The STAR framework is composed from four main components: Introduction, Puzzles, Resolution, and Debriefing (Fig. 1). It should be noted that this framework was designed to be suitable especially for storytelling within games, in which the story is similar to a detective story. The reason behind this is the enduring popularity of investigative-style games (CSI/ Phoenix Wright / Heavy Rain) coupled with the natural opportunities such games offer for puzzle structures. From a pedagogical view we follow Problem Based Learning (PBL) [11]. Although there is no consensus on how pedagogical approaches are to be used in educational games, PBL has been successfully used in several games [12-13].

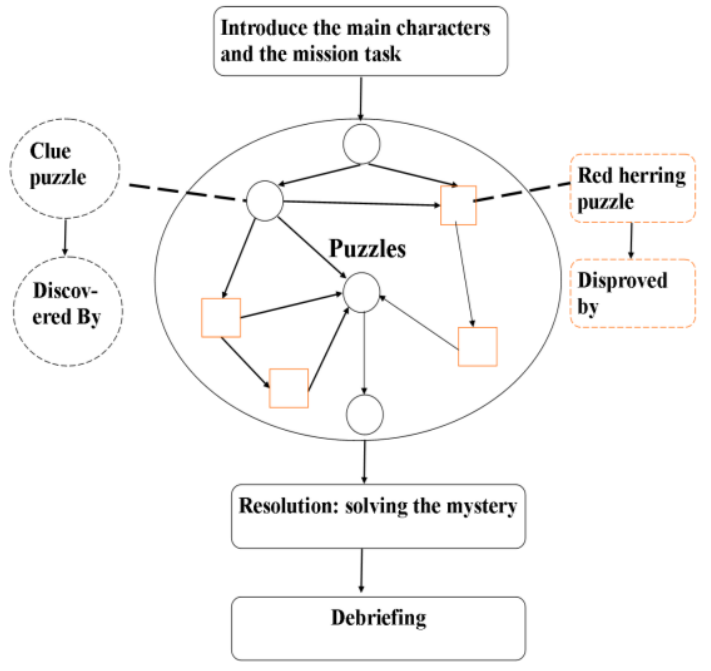

Fig. 1. Story structure

In PBL the user is introduced to the problem before the learning occurs and the process through which the problem is understood and solved drives learning [14]. Learners generate hypotheses as to how they may solve the problem, and through self-directed research evaluate their hypothesis on the basis of evidence. The problem is then solved or, if their hypothesis is disproven, a new hypothesis is generated. This process maps well to quest structures found in story-based games [15]. When referring to the problem to be solved we will use from here on the term mission task as this term incorporates the idea of integrating the "problem" with the story's "mission".

The player will visit various locations during their investigation to solve the mission task. Each location has a number of items that the player can find by clicking 
on them. Each item (excluding those put in as false clues) either corroborates or contradicts a statement that has been or will be made by characters the player meets.

Each location also has one or more characters present. By talking to these characters, the player finds out background information on the puzzle at hand. Some characters will suggest theories as to the cause or source of the problem. The player will find items (or other evidence such as quotes from other characters) that corroborate or contradict these statements. By presenting the appropriate evidence at the correct time, the player furthers the dialogue and receives further information about the problem. The player must pay attention to what characters are saying, understand their point of view and find evidence relevant to their statements.

\subsection{Introduction}

The first part of each story introduces the main characters, and the mission task. The characters used in the storytelling are designed to resonate with the target group for which the story is intended.

\subsection{Puzzles}

The second component of our framework is composing the LOs as a set of puzzles that have to be untangled to solve the mystery presented in the Introduction. This part of the game allows the player to explore the environment. According to [16] the environments in which the students can actively explore lead to better learning. Moreover, this part of the story teaches the educational element of the games, the Learning Objectives (LOs). It is important to have the educational content integrated in the story, as otherwise the learner ignores factual content [10].

The puzzles require the player to read and understand the statements made by characters and then find evidence that corroborates or contradict these statements. The player must understand the content in order to be able to identify the current clue to prove or disprove each statement. If the puzzle to be solved lead to approving the statement we will call it from here on a clue puzzle, whereas if the puzzle lead the player to disprove a statement we call it a red herring puzzle. The puzzles are usually relevant to solving the mystery. Not all the puzzles are mandatory for solving the mystery and the player can explore different paths in the game.

The LOs are integrated in the puzzles, with each of the puzzle having one or more LOs (Fig. 2-a). Due to the non-linearity of the story, and because the player can choose different paths, the same LO could occur in different puzzles. Another reason to repeat one LO in multiple puzzles is that it affords reinforcement of important LOs. In this framework clue puzzles are puzzles that are mandatory for the player to solve, while red herring puzzle can be mandatory or not, leading to the interactivity of the story. It is important that each path the player can take through the game has to cover the mandatory LOs for which the story was designed, therefore puzzles can be designed to act as 'gates' where the player must complete that puzzle before continuing to a further part of the mission. It is the designer's responsibility to ensure that the all the LO are covered by the mandatory puzzles, one way to do it is by using 
a table similar to the one presented in Fig. 2 which will cover all the clue puzzles and the mandatory red herring puzzles.

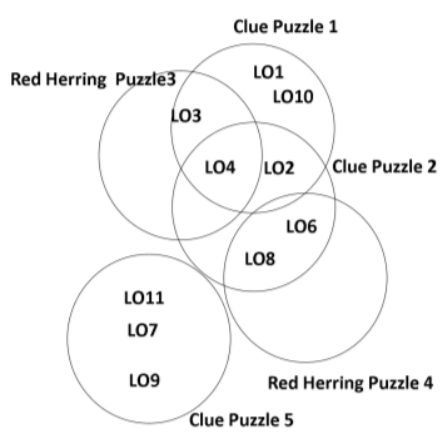

a)

\begin{tabular}{|c|c|c|c|c|c|}
\hline & P1 & P 2 & $\cdots$ & P m & LO Covered \\
\hline LO 1 & $\mathrm{x}$ & $\mathrm{x}$ & & & $\mathrm{v}$ \\
\hline LO 2 & $\mathrm{x}$ & & & & $\mathrm{v}$ \\
\hline LO 3 & & $\mathrm{x}$ & & & $\mathrm{v}$ \\
\hline$\ldots$ & & & & & $\mathrm{v}$ \\
\hline LO n & & & & $\mathrm{x}$ & $\mathrm{v}$ \\
\hline
\end{tabular}

b)

Fig. 2. a) LO b) LO Covered by the Mandatory Puzzles

\subsection{Resolution}

This is the part of the story where the player reaches to a resolution regarding the mystery: who was the guilty party, what the problem was etc. It basically represents the point where the player solved the "mission task" defined in the introduction section.

\subsection{Debriefing}

In the debriefing phase the player explains the steps they took to solve the mystery. The player re-presents the solution to the mission task and explains the steps required to reach the solution (based on the puzzle visited). From an educational point of view this section acts as a summative assessment of the collection of educational content hence reinforcing the LOs taught and allowing measurement of the player's knowledge.

\section{Case Study}

Considering that we start with the following list of LO:

LO 1: Microbes can be found on our food and can transfer to humans

LO 2: Cooking food properly can kill harmful microbes

LO 3: Sometimes microbes can make us sick

LO 4: Infection can be spread through unclean hands

LO 5: Hand washing can prevent the spread of infection

LO 6: When to wash your hands

LO 7: Why to wash your hands 
LO 8: Microbes can pass between different food products

LO 9: Separate utensils should be used for meat and vegetable products

LO 10: Wash your hands after using the toilet, even after 'just' a pee

LO 11: Don't spread your bad microbes to others

Starting with these LOs we created the story of the Bad Bacteria at BBQ/GHD (Global Handwashing Day) Game which we will use for exemplification purposes. The game was designed using the STAR framework. The game is an adventure game, where the player adopts the role of an investigator that travels to countries across the world to investigate and solve microbial problems.

\subsection{Introduction}

During the introduction the player finds that they are a junior detective working for an investigation agency. Moreover, the main characters: Big C and Alyx are introduced here, whereas the rest of characters are introduced as the story unfolds (Fig. 3). Alyx is a young scientist who is assigned to be your partner at the beginning of the first mission. She accompanies you wherever you go and is used in the game to further narrative where required. Alyx is also the character we use to give hints or prompt the player when we think s/he is stuck. We also use Alyx to carry out tests in the portable lab if needed.

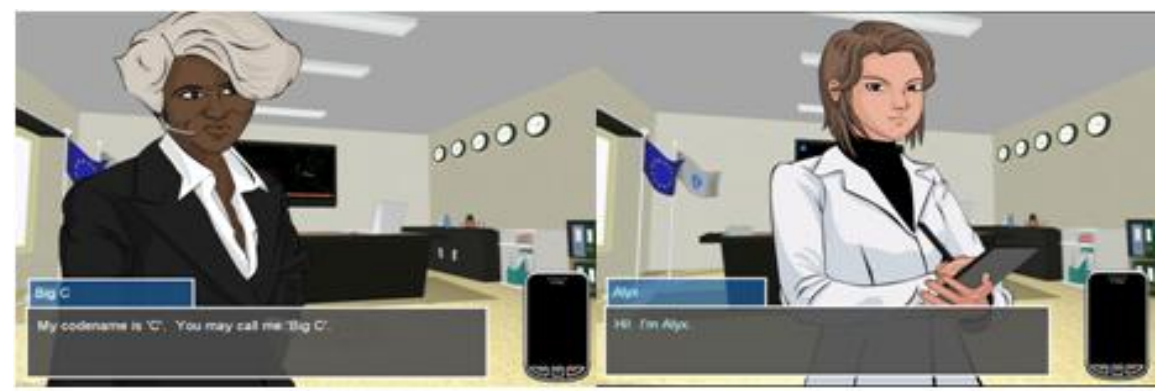

Fig. 3. Big C and Alyx

Big $\mathrm{C}$ is the head of the investigation agency. Most of the characters in the game were designed to appeal to the age group of the children and be a "cool" character they easily associate with (based on our focus groups) while Big $\mathrm{C}$ who is the boss and is older, represents authority and senior expertise - her role is to assign the mission task in the Introduction and debrief the player in the Debriefing session. After the scene is set, Big C introduces the mission task, Hugh Gaego, a famous actor who earlier this evening won the Best Male Actor award at the International Film Awards, has been taken ill and a possible poisoning has to be investigated by the player.

\subsection{Puzzles}

There are currently six puzzles integrated in the game. We worked with a group of experts in the area to determine which specific LO to include based on the European 
curriculum [17]. Although the same LO appears multiple times, it is presented each time in a different context so that the story will not be boring for players, moreover all the $\mathrm{LO}$ are covered by at least one mandatory puzzle (Fig. 2).

There are four locations in which the puzzles take place: the barbeque, the bathroom, the kitchen, and the laboratory. Additionally the agency headquarters are introduced at the beginning. The barbeque is happening at Hugh's mansion and has been thrown to celebrate his success. It is the last place where Hugh eats before he gets sick. The player will meet Hugh in the bathroom due to the fact that he is continuously ill. For meeting the chef (the initial suspect in this case) the player would have to travel to the kitchen. The laboratory is the place where the data analysis takes place.

The barbeque, the toilet and the kitchen contain various items which the player can collect by clicking on them. For example the following items and the associated evidence can be found and collected at the barbeque:

1. Punchbowl

a. No unusual microbe activity.

2. Hugh's plate of leftovers

a. Shows microbe activity on the salad and not so much on the cooked chicken

b. When tested in the laboratory e-coli bacteria and the vomiting virus are found

c. This is unusual for salad.

3. Salad bowl

a. Shows some signs of unusual microbe activity.

4. Hand sample from bodyguard

a. As collected on an agar plate

b. Shows high level of microbe activity.

Below we present the puzzle existent in the game.

\section{Food poisoning from chicken - Red Herring Puzzle.}

Learning Objectives: LO 1, LO2, and LO3.

Puzzle Setup:

1. Hugh's bodyguard suggests that the kitchen is unhygienic

2. Player finds a raw-meat chopping board in the kitchen that is covered in microbes.

Disproved by:

1. Player will find plate the Hugh has been eating from containing the chicken and salad and take it to the lab to test it

2. Chicken is cooked correctly

3. Microbial levels in chicken are normal

4. The security guard mentions that the other guests who also ate chicken are fine 


\section{Food poisoning from salad - Clue Puzzle}

Learning Objectives: LO 1, LO2, and LO3.

Puzzle Setup:

1. Player has disproven the hypothesis that the cooked chicken was the source of bacterial transfer (see above)

2. The other item of food on Hugh's plate was salad.

Discovered by:

1. Player collects salad and take it to the lab to test it

2. Salad returns positive for e-coli and vomiting virus

3. This is unusual as you would not expect to find these on salad.

\section{Contamination via chopping board - Red Herring Puzzle}

Learning Objectives: LO 1, LO 9, and LO 10.

Puzzle Setup:

1. Hugh's bodyguard has suggested that poor kitchen hygiene could be the source of Hugh's illness

2. Player has found a raw-meat chopping board that has a high concentration of bacteria

3. The player cannot see a vegetable chopping board. Thus, if the same chopping board were used for salad as raw meat, bacteria could transfer.

Disproved by:

1. Player tests chopping board which has been used to prepare chicken but only has expected campylobacter which is usually contained in uncooked meat but the microbe that has infected Hugh is not found

2. The cooked chicken from Hugh's plate does not have the campylobacter suggesting that it has been cooked correctly

3. Chef insists the veggie board is in dishwasher.

\section{Bad kitchen hand washing - Red Herring Puzzle}

Learning Objectives: LO 4, LO5, LO 6, and LO 7

Puzzle Setup:

1. The player has identified that there are unexpected bacteria and vomiting viruses in the salad that Hugh ate (see above)

2. The player has ruled out contamination from chopping boards (see above)

3. The player is trying to find out how the microbes were transferred to the salad and since the Chef handles the raw meat, he could be the source.

Disproved by:

1. Player taking a swab of Chef's hands and take it to the lab to test it

2. Chef's hands have mostly normal good microbes 
3. Only very small (inconsequential) quantity of e-coli implying that he does wash his hands but may have come in contact with a source.

\section{Contamination poor post-loo hand hygiene by Chef- Red Herring Puzzle}

Learning Objectives: LO 4.

Puzzle Setup:

1. Since the bacteria that was found on the salad was not found on the raw-meat chopping board, it is possible that the chef transferred other bacteria obtained through poor bathroom hygiene to Hugh's food.

Disproved by:

1. Chef's handprint in agar is tested in the laboratory and shows too few microbes for the Chef to be source.

\section{Contamination poor post-loo hand hygiene by Hugh's bodyguard - Clue Puzzle} Learning Objectives: LO 4, LO 5, LO 6, LO 7, LO 10, and LO 11.

Puzzle Setup:

1. Having ruled out bad kitchen hygiene and also ruled out the Chef as a likely source of 'fecal-oral' bacterial contamination via salad, the player seeks alternative sources.

Discovered by:

1. A handprint on the bathroom door shows the same bacteria as was found on Hugh's salad

2. The bodyguard's fingerprint in agar is tested and matches the handprint found on the bathroom door.

\subsection{Resolution}

At this point the player solved the mission task. In this case, the bodyguard poor

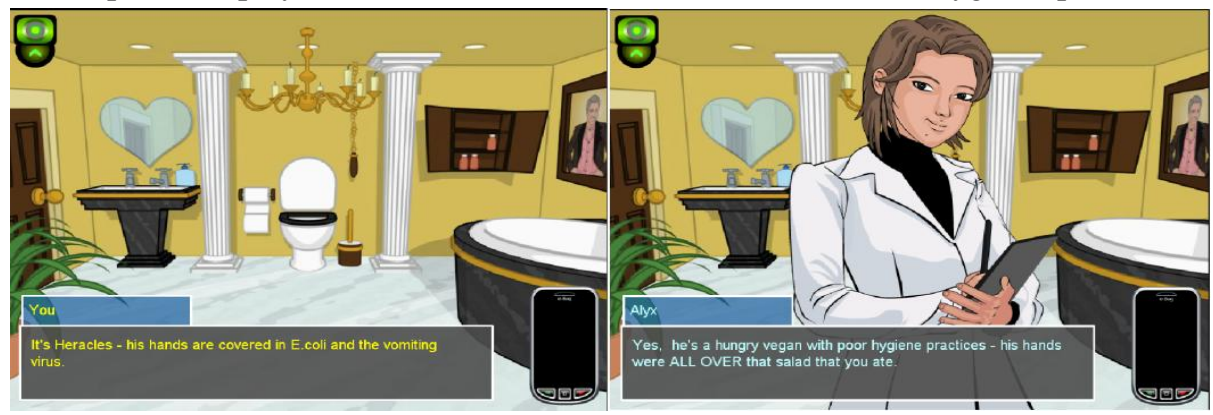

Fig. 4. The guilty person is found 
bathroom hygiene, penchant for salad (he is a vegan) and lack of food hygiene habits (he used his fingers to nibble on the party salad) - all lead to him accidentally poison Hugh. The player returns to Hugh to tell him the results of the investigation (Fig. 4).

\subsection{Debriefing}

After the investigation is over, the player returns to Big C for debriefing. Through dialogue, Big $\mathrm{C}$ asks questions of the player that provide the opportunity for the player to expand upon the puzzles they followed and describe what was discovered. While explaining the findings of the investigation the player repeats some of the LOs covered in the story.

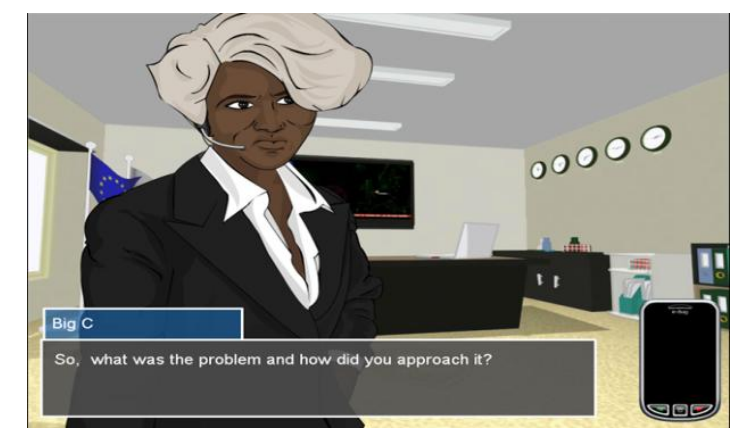

Fig. 5. Debriefing

\section{Evaluation, Lesson Learnt and Future Work}

Three games were created using the STAR framework, with the aim of teaching health and hygiene related issues:

1. Bad Bacteria at BBQ/GHD Game: focuses on hand and food-preparation hygiene

2. When Bugs go Wild: focuses on responsible antibiotic use

3. Gambling Never Pays: also focuses on responsible antibiotic use but following slightly different learning objectives

A small sample study was initially performed to assess the games effectives in conveying the LOs [18]. For GHD Game a second study has been done with 145 participants and the results of the evaluation show statistically significant knowledge gain as a result of playing the game.

The players enjoyed the games but they took too long to play and some players quit the game before finishing [18]. Although it is not something unusual, and has been reported in previous other studies [19-20], the games have been shortened. For example the GHD Game, presented here, had originally ten puzzles. Due to the nonlinearity of the story it was possible to simplify the game without having to rethink the narrative and plot. This shows the flexibility of the STAR framework. In future 
work we will explore different techniques to keep players engaged, such as to improve the competition element, explore multiplayer options and to introduce a social aspect.

We also noticed that players participating in the study do not enjoy taking the pen and paper pre and especially post tests after playing the games. Following this observation, we are currently working to have the evaluation of the LOs seamlessly integrated in the game, with the aim of reducing the inconvenience of the pre and post test pen and paper evaluations.

We are also planning to add an adaptive dimension to the story, in which the game changes not only based on the player actions but also on his knowledge level. It would be also interesting to explore if there is any difference in term of conveying the educational message between the red herring puzzles and clue puzzles.

\section{Conclusions}

This research presented a novel framework, STorytelling for educAtional inteRventions (STAR), for designing stories for games intended for educational interventions. The framework integrates a given set of LOs tightly with narrative and puzzle design to teach through the story. The framework has four main components: Introduction, Puzzles, Resolution, and Debriefing. This framework has been successfully used in creation of three games aimed to teach important concepts related to health, one of which was illustrated in this paper as case study. The results of the games evaluation show that the games are both enjoyable and efficient in conveying the educational message. Although this paper presents the story design rather than the implementation, it should be noted that the story success depends on different factors such as the implementation of the game, the artwork used, and pedagogy etc.

Acknowledgement: DGSANCO for funding e-Bug and projects partners for their contribution, Christina Daly and Dr. Gabriel-Miro Muntean for helping with the evaluation.

\section{References}

1. Hodhod, R., Cairns, P., Kudenko, D.: Innovative Integrated Architecture for Educational Games: Challenges and Merits. In V. Z. Pan, A. D. Cheok, W. Müller, X. Yang (Eds.). Trans. on Edutainment, pp. 1-34. Springer-Verlag, Berlin, Heidelberg (2011)

2. O'Neil, H.F., Perez, R.S.: Computer Games and Team and Individual Learning. Amsterdam, Elsevier (2008)

3. Bergström, K.: Framing Storytelling with Games. In M. Si, D. Thue, E. André, J. C. Lester, J. Tanenbaum (eds.) ICIDIS 2011, pp. 170-181. Springer-Verlag, Berlin, Heidelberg, 170181. (2011)

4. Smith, G., Anderson, R., Kopleck, B., Lindblad, Z., L. Scott, A. Wardell, J. Whitehead, M. Mateas. Situating Quests: Design Patterns for Quest and Level Design in Role-Playing 
Games. In M. Si, D. Thue, E. André, J. C. Lester, J. Tanenbaum (eds.) ICIDIS 2011, pp. 326-329. Springer-Verlag, Berlin (2011)

5. Szilas, N., Axelrad, M., Richle, U.: Propositions for Innovative Forms of Digital Interactive Storytelling Based on Narrative Theories and Practices. In V. Z. Pan, A. D. Cheok, W. Müller, X. Yang (Eds.). Trans. on Edutainment, LNCS 7145, pp. 161--179. Springer, Heidelberg (2012)

6. Murray, J.H.: Why Paris Needs Hector and Lancelot Needs Mordred: Using Traditional Narrative Roles and Functions for Dramatic Compression in Interactive Narrative. In M. Si, D. Thue, E. André, J. C. Lester, J. Tanenbaum (eds.) ICIDIS 2011, pp. 13-24. SpringerVerlag, Berlin, Heidelberg (2011)

7. Spires, H. A., Rowe, J., Mott, B., Lester, J.: Problem Solving and Game-Based Learning: Effects of Middle Grade Students' Hypothesis Testing Strategies on Learning Outcomes. Journal of Educational Computing Research, 44(4), 453-472 (2011)

8. Mehta, M., Corradini, A., Ontannon, S., Henrichsen. P.J. Textual vs. Graphical Interaction in an Interactive Fiction Game. In R. Aylett, M. Y. Lim, S. Louchart, P. Petta, M. Riedl (eds.) ICIDIS 2010, pp. 228-231. Springer-Verlag, Berlin, Heidelberg (2010)

9. Adams, D. M., Mayer, R E., MacNamara, A, Koenig, A, Wainess, R.: Narrative Games for Learning: Testing the Discovery and Narrative Hypotheses. J. of Educational Psychology, 104(1), 235-249 (2012)

10. Wolfe, M. B. W., Mienko, J. A.: Learning and Memory of Factual Content from Narrative and Expository Text. British Journal of Educational Psychology, 77, 541-564 (2007)

11. Savery, J. R., Duffy, T. M.: Problem Based Learning: An Instructional Model and its Constructivist Framework. Educational Technology, 35, 31-38 (1995)

12.Sancho, P., Moreno-Ger, P., Fuentes-Fernández, R., Fernández-Manjón, B.: Adaptive Role Playing Games: An Immersive Approach for Problem Based Learning. Educational Technology \& Society, 12 (4), 110-124 (2009)

13. Cheng, Y.-M., Kuo, S.-H., Lou, S.-J., Shih, R.-C.: The Construction of an Online Competitive Game-Based Learning System for Junior High School Students. The Turkish Online Journal of Educational Technology, 11(2) (2012)

14. Barrows, H. S., Tamblyn, R. M.: Problem-based Learning: An approach to Medical Education. New York: Springer. (1980)

15.Farrell, D., Kostkova, P., Lecky, D., McNulty, C.: Teaching Children Hygiene Using Problem Based Learning: The Story Telling Approach to Games Based Learning. International Conference on Web-based Learning Second Workshop on Story-Telling and Educational Games (2009)

16. Mayer, R. E.: Applying the science of learning. Upper Saddle River, NJ: Pearson (2010).

17. Donna M Lecky, Cliodna A. M., McNulty et al. What are School Children in Europe being Taught about Hygiene and Antibiotic Use?, v13-21. J. Antimicrob. Chemother. 66 Suppl 5 (suppl_5) (2011)

18.Kostkova, P.: Seamless Evaluation of Interactive Digital Storytelling games: Edugames4All, In eHealth, Springer Lecture Notes of the Institute for Computer Sciences, SocialInformatics and Telecommunications Engineering LNICST 91, 80-84. (2012)

19.Snow, B.: Why most People don't Finish Video Games, http://articles.cnn.com/2011-08$\underline{17 / \text { tech/finishing.videogames.snow_1_red-dead-redemption-entertainment-software- }}$ association-avid-gamers?_s=PM:TECH

20.Schoenau-Fog, H. Hooked! --- Evaluating Engagement as Continuation Desire in Interactive Narratives. In M. Si, D. Thue, E. André, J. C. Lester, J. Tanenbaum (eds.) ICIDIS 2011, pp. 219-230. Springer-Verlag, Berlin, Heidelberg. 